\title{
Applications of hydroxy acids: classification, mechanisms, and photoactivity
}

This article was published in the following Dove Press journal:

Clinical, Cosmetic and Investigational Dermatology

23 November 2010

Number of times this article has been viewed

\author{
Andrija Kornhauser' \\ Sergio G Coelho \\ Vincent J Hearing ${ }^{2}$ \\ 'US Food and Drug Administration \\ [retired], Annandale, VA, USA; \\ ${ }^{2}$ Laboratory of Cell Biology, National \\ Cancer Institute, National Institutes \\ of Health, Bethesda, MD, USA
}

\begin{abstract}
Hydroxy acids (HAs) represent a class of compounds which have been widely used in a number of cosmetic and therapeutic formulations in order to achieve a variety of beneficial effects for the skin. We review and discuss the most frequently used classes of these compounds, such as $\alpha$-hydroxy acids, $\beta$-hydroxy acids, polyhydroxy acids, and bionic acids, and describe their applications as cosmetic and therapeutic agents. Special emphasis is devoted to the safety evaluation of these formulations, in particular on the effects of their prolonged use on sun-exposed skin. Furthermore, we summarize the very limited number of studies dealing with the modifications evoked by topical application of products containing HAs on photocarcinogenesis. In spite of the large number of reports on the cosmetic and clinical effects of HAs, their biological mechanism(s) of action still require more clarification. Some of these mechanisms are discussed in this article along with important findings on the effect of HAs on melanogenesis and on tanning. We also emphasize the important contribution of cosmetic vehicles in these types of studies. Thus, HAs play an important role in cosmetic formulations, as well as in many dermatologic applications, such as in treating photoaging, acne, ichthyosis, rosacea, pigmentation disorders, and psoriasis.
\end{abstract}

Keywords: hydroxy acids, glycolic acid, salicylic acid, UV, erythema, cosmetics

\section{Introduction}

Formulations containing hydroxy acids (HAs) have been used in clinical practice for decades to treat a variety of skin conditions. The most prominent representatives in this class of compounds are glycolic acid, lactic acid, and salicylic acid (SA) (Figure 1). HA have transformed skin care since their introduction to dermatology. ${ }^{1}$ They have been used, typically in concentrations ranging from $2 \%$ to $70 \%$, to treat acne, ichthyosis, keratoses, warts, psoriasis, photoaged skin, and other disorders. ${ }^{2}$ In the last three decades, $\alpha$-hydroxy acids ( $\alpha$ HAs) have been widely incorporated into a variety of cosmetic products for daily use over long periods of time. ${ }^{3}$ Currently, glycolic acid, lactic acid, and SA are commonly used in cosmetics. One of the most cited beneficial effects of HAs is the reported improvement of photoaged skin. These improvements have been measured as decreases in roughness, discoloration, solar keratoses, and overall pigmentation and also as increased density of collagen and improved quality of elastic fibers. ${ }^{4}$ The antiaging effects of HA have become a prominent factor in cosmetic dermatology, leading to the proliferation of HA-containing cosmetic products and skin care systems. ${ }^{4}$ More recent research in this field led to the discovery of polyhydroxy acids (PHAs) and aldobionic acids (BAs) (Figure 1), which are presently widely used in cosmetic and dermatologic applications. ${ }^{5}$ Many
Correspondence: Andrija Kornhause 4517 Pinecrest Hgts Dr, Annandale, VA 22003, USA

Tel +l 703 94| 422I

Email akornhause@aol.com 
<smiles>O=C(O)CO</smiles>

Glycolic acid<smiles>O=C(O)c1ccccc1O</smiles>

Salicylic acid<smiles>O=C(O)[C@@H](O)[C@@H](O)[C@@H](O)CO[C@@H]1O[C@H](CO)[C@@H](O)[C@H](O)[C@H]1O</smiles>

Lactobionic acid<smiles>C[C@H](O)C(=O)O</smiles>

Lactic acid<smiles>O=C(O)CC(O)C(=O)O</smiles>

Malic acid<smiles>O=C(O)C(CO)c1ccccc1</smiles>

Tropic acid<smiles>O=C1O[C@H](CO)[C@@H](O)[C@H](O)[C@H]1O</smiles>

Gluconolactone

Figure I Structures of various compounds discussed

preparations that contain HAs are both exfoliants and moisturizers. In low concentrations $(4 \%-10 \%)$, they are ubiquitous components of nonprescription creams and lotions that are promoted as being effective for ameliorating skin aging. In high concentrations $(>20 \%)$, these preparations are used as chemical 'peels' to treat calluses, keratoses, acne, psoriasis, and photoaging.

This review is intended to provide a summary of the most frequently used classes of HAs, to describe their applications as cosmetic and therapeutic agents, and to discuss their biologic mechanisms of action. We initially discuss the types/classes of HAs, then consider their safety evaluation, implications to photocarcinogenesis, mechanisms of their biological actions, and their effects on pigmentation.

\section{Types/classes of HAs $\alpha \mathrm{HAs}$}

$\alpha H A s$ are carboxylic acids with one hydroxyl group attached to the $\alpha$-position of the carboxyl group. The simplest representative of $\alpha \mathrm{HA}$ is glycolic acid (chemical name, hydroxyacetic acid), which was the first of this class of compounds to be introduced into skin care products. ${ }^{3}$ Lactic acid, with optimal biologic activity in its L-form, is also used in various topical formulations to exfoliate the skin and also to provide antiaging properties. ${ }^{6}$

\section{PHAs}

A new generation of $\alpha H A s$, called PHAs and polyhydroxy bionic acids (PHBAs), provide effects similar to $\alpha H A s$ but with less irritation responses. PHAs, such as lactobionic acid (Figure 1), are carboxylic acids with two or more hydroxyl groups attached to carbon atoms or an alicyclic chain. It is essential that at least one hydroxyl group be attached to the $\alpha$-position. Attaching a sugar molecule to the PHA structure results in a polysaccharide known as bionic acid. Multiple skin benefits have been proven for the PHAs and PHBAs, making them ideal ingredients for use in dermatologic and cosmetic procedures. ${ }^{5}$ 


\section{$\beta$-Hydroxy acids}

$\beta$-Hydroxy acids ( $\beta$ HAs) are carboxylic acids having one hydroxyl group attached to the $\beta$-position of the carboxyl group. The most common $\beta \mathrm{HA}$ is $\beta$-hydroxybutanoic acid. A lipid soluble $\beta \mathrm{HA}$ is tropic acid (2-phenyl-3-hydroxypropanoic acid) (Figure 1). Some $\beta$ HAs are also considered $\alpha H A s$ as they contain a hydroxyl group in the $\alpha$-position to one carboxyl group and in the $\beta$-position to the other carboxyl group. Malic acid and citric acid are prominent representatives in this category (Figure 1). Citric acid is widely used in topical formulations as an antioxidant, and its antiaging benefits are well established.?

\section{SA}

In the cosmetic and dermatologic literature, $\mathrm{SA}$ is often described as a $\beta \mathrm{HA}$, but that classification is incorrect. ${ }^{8}$ In SA, both the hydroxyl and the carboxyl groups are directly attached to an aromatic benzene ring and both exhibit acidic properties. In contrast, the hydroxy groups in $\alpha H A s, \beta H A s$, and PHAs are neutral under the conditions used in clinical and cosmetic settings. On the basis of knowledge to date, SA does not function physiologically or therapeutically as a $\beta$ HA. SA is used in cosmetic formulations for a variety of applications, more specifically, it is fat soluble, which makes it useful in subjects with oily skin.

\section{Safety evaluation of HAs}

Questions have been raised about the safety of prolonged use of HA-containing products on sun-exposed skin. A number of clinical studies have reported that topical application of glycolic acid can increase sensitivity of the skin to solar-simulated radiation (SSR). ${ }^{9}$ Most of these studies used vehicles which differ significantly from those used in cosmetic products. Furthermore, only a single cutaneous biomarker for UVinduced damage was usually measured. In the few cases in which multiple endpoints were used, they were not evaluated in the same subject. ${ }^{10}$ In a previous study using 16 subjects, we reported that $10 \%$ glycolic acid, applied 6 days/week for 4 weeks, enhanced the sensitivity to UVB radiation, measured as increased sunburn cell formation, and also lowered the minimal erythemal dose (MED). ${ }^{10}$ In another cohort group $(n=13)$, levels of cyclobutane pyrimidine dimers were elevated in $\alpha H A-t r e a t e d ~ s k i n$, but not to a statistically significant level. We also found that the enhanced photosensitivity was reversed within a week of terminating the treatments. Another of our collaborative studies was designed to generate new data on the effects of topically applied glycolic acid and SA on the sensitivity of human skin to SSR. ${ }^{9}$ A total of 14 subjects with skin types II to III were involved in that study. Biomarkers measured were erythema, cyclobutane pyrimidine dimers, and sunburn cells (apoptotic keratinocytes). The protocol was designed to allow us to measure all of the above biomarkers in each subject, with the vehicle-treated skin of each subject serving as his/her own control. We found that glycolic acid $(10 \%)$ increased the SSR sensitivity of human skin, whereas SA $(2 \%)$ did not. The photosensitizing effects of glycolic acid were small, but they may be an important factor for public health, considering that HA-containing products are being used by a large segment of the population for many years and in many cases over a lifetime.

In 1998, the Cosmetic Ingredient Review (CIR) Expert Panel evaluated the available evidence and concluded that $\alpha \mathrm{HA}$ ingredients are not mutagenic or carcinogenic, are not reproductive or developmental toxins, and are not skin sensitizers. ${ }^{11}$ To reduce the risk of skin irritation, the CIR Panel recommended limitations on the concentration of $\alpha \mathrm{HAs}(<10 \%)$ and the $\mathrm{pH}$ (at or above 3.5) of cosmetic products containing $\alpha$ HAs. In addition, the CIR Panel recommended that $\alpha H A$-containing products should be formulated to avoid enhancing sun sensitivity and that consumers should be advised to use daily sun protection. ${ }^{11} \mathrm{SA}$ is added to cosmetic products at concentrations $<3 \% .{ }^{12}$ The CIR Panel similarly recommended that effects on skin sensitivity to sunlight be considered in the formulation and use of products containing SA and salicylates. ${ }^{12}$

\section{Photocarcinogenesis}

The importance of solar UVR exposure in the etiology of cutaneous tumorigenesis has long been firmly established. The nature and magnitude of the modifications evoked by topical application of various cosmetic/therapeutic products are much less understood. An extensive project, sponsored by the National Toxicology Program, of the photocarcinogenic potential of HAs in SKH-1 mice was recently completed. ${ }^{13}$ That study was designed to test the hypothesis that topical application of creams containing glycolic acid (4\% or $10 \%$; pH 3.5) or SA ( $2 \%$ or $4 \%$; pH 3.5) on mice could enhance the rate of skin cancers induced by SSR. In order to test this hypothesis, mice were treated 5 days/week with each cream, followed by exposure to SSR. This treatment was continued for 40 weeks, followed by 12 weeks of no additional treatment prior to sacrifice of the animals. Tumors induced under the described experimental conditions in mice were squamous cell papillomas, carcinomas in situ, and squamous cell carcinomas. Taking into consideration the survival data, time to tumor data, and the pathology results, glycolic acid did not alter the photocarcinogenesis induced by SSR, while 
SA at $4 \%$ showed a photoprotective activity, reducing the carcinogenicity of SSR.

The role of the vehicle control cream was also investigated in that study. Surprisingly, a SSR dose-dependent increase in the formation of skin neoplasms was observed in mice treated with the control cream compared with mice not treated with the cream. These results indicate that the control cream enhanced the photocarcinogenesis of SSR in the SKH-1 mice under these test conditions. They further indicate that the proper control groups for statistical comparison of the effects of glycolic acid or SA are mice that received the control cream, and not the mice untreated with a cream. ${ }^{13}$

In addition to the described photocarcinogenesis study, the same investigators examined the acute proliferative effects of glycolic acid (10\%; $\mathrm{pH} 3.5-4.0)$ and SA (4\% or $10 \%$; $\mathrm{pH} 3.5-4.0$ ) on cell proliferation in the epidermis of SKH-1 female mice, using BrdU incorporation as a marker of epidermal proliferation. ${ }^{14}$ They found a dose-dependent increase in epidermal proliferation in mice treated with either glycolic or SA. A similar response was observed in the epidermal thickness of mice treated with SA, but not with glycolic acid. Their study demonstrates that topical administration of glycolic acid or SA can affect cellular proliferation in mouse epidermis. In a second study, the same authors described the effect of the corresponding formulations of glycolic acid and SA on the edemal response to SSR in female SKH-1 mice. ${ }^{15}$ They found that the use of creams containing glycolic or SA for up to 6 weeks does not increase the susceptibility to an edemal response caused by exposure to SSR. The effects of the long-term use of such creams in combination with exposure to SSR are yet to be established. It is noteworthy that the vehicle and test materials used in the above described chronic and acute studies in mice had the same composition and origin as those used in our clinical study. ${ }^{9}$ Furthermore, the spectral distribution of the SSR sources was the same in all these studies. This allows a comparison between results on HA-induced changes in acute responses to SSR in humans and their corresponding responses in an animal model.

In 2001, Hong et al published a study on the photocarcinogenic potential of topically applied glycolic acid in SKH-1 mice. They found that glycolic acid had an inhibitory effect against UV-induced skin tumor development in this system. ${ }^{16}$ The protective effect of glycolic acid was a $20 \%$ reduction of skin tumor incidence, a 55\% reduction in tumor multiplicity (average number of tumors/mouse), and a $47 \%$ decrease in the number of large tumors $(>2 \mathrm{~mm})$. Glycolic acid also delayed the first appearance of tumor formation by about 3 weeks. There are, however, some important differences between the experimental designs described by Hong et al compared to the protocol applied in the National Toxicology Program study, which greatly limit comparison of the results from the two studies. In the study of Hong et al glycolic acid was dissolved in polyethylene glycol and was applied at a large dose of $8 \mathrm{mg} / \mathrm{cm}^{2}$ (the authors called it a therapeutic dose) on the skin. The UV irradiation was carried out with a UV lamp source, not with SSR. Furthermore, the omission of a vehicle control group in the study of Hong et al raises additional concern.

The effects of the topical control cream have been described by several other investigators. It has been known for some time now that topically applied formulations such as emollients and moisturizers may influence the optical properties of the skin and therefore may influence the optical effectiveness of UV exposures. Moisturizing creams and ointments used for the prevention and treatment of dry skin are generally tested for safety by determining their irritant and sensitizing (immunological response) activities. These preparations are generally not tested for their carcinogenic or cocarcinogenic properties. A significant contribution to this field was recently published by Lu et al. ${ }^{17}$ They developed an animal model (SKH-1 mice) that mimics sunlightinduced skin cancers in humans who receive heavy exposure to sunlight early in life, but develop skin cancers later in life in the absence of subsequent heavy sunlight exposure. To accomplish this, SKH-1 mice were treated twice weekly with UVB for 3 months and after that they were no longer exposed to UVB. These mice do not have tumors, but they do have a high risk for developing skin tumors over the next several months even in the absence of further UVB irradiation (high-risk mice). The authors tested four commercially available and widely used moisturizing creams and found that all of them had tumorigenic activity when applied topically to high-risk mice. Histological characterization of the tumors was not done in their study. Prior to the report by Lu et al little attention was paid to the possibility of a post-UV effect by these seemingly innocuous formulations. The study by Lu et al raises the question of whether these agents belong to a list of more active compounds analogous to tumor promoters in two-stage carcinogenesis, in which UVB is the initiator. ${ }^{18}$ It has to be emphasized that this study was performed in mice, and therefore, its significance for humans remains unproven. It should be noted that the stratum corneum plays an important role in photoprotection, and since many of the HAs have exfoliative effects, that might at least in part play a role in changes in photoprotection. 


\section{Mechanism of biological actions of HAs}

Although there are many reports about the cosmetic and clinical effects of HAs, their biological mechanism(s) of action still needs to be clarified. Several key studies relevant to this important issue will be discussed here.

\section{$\alpha \mathrm{HAs}$}

Based on analysis of available experimental and clinical data, Wang proposed a mechanism of action for $\alpha$ HAs topically applied to the skin. ${ }^{19}$ That analysis was based on data regarding the chelation properties of $\alpha \mathrm{HAs}^{20}$ and the fact that calcium ions are known to play a crucial role in cutaneous cellular adhesions. ${ }^{21}$ Wang proposed a theory that $\alpha \mathrm{HAs}$ reduce the $\mathrm{Ca}$ ion concentration in the epidermis and remove $\mathrm{Ca}$ ions from the cell adhesions by chelation. This process would cause a loss of $\mathrm{Ca}$ ions from the cadherins of the desmosomes and adherens junctions. When $\mathrm{Ca}$ is decreased, cellular adhesions are disrupted and exfoliation takes place. Wang further proposed that the decrease of $\mathrm{Ca}$ ions in the epidermis also tends to promote cell growth and to retard cell differentiation, and therefore caution should be taken regarding excessive and chronic use of these compounds.

Okano et al conducted a study that investigated the effects of glycolic acid on the dermal matrix metabolism of keratinocytes and fibroblasts using in vitro and ex vivo (human skin biopsies) systems. ${ }^{22}$ That study showed that glycolic acid not only directly accelerates collagen synthesis by fibroblasts, but that it also modulates matrix degradation and collagen synthesis through keratinocyte-released cytokines. Their experiments confirmed that IL-1a is one of the primary mediators regulating matrix degradation that are released from keratinocytes after glycolic acid treatment. On the basis of their findings, the authors suggest that glycolic acid contributes to the recovery of photodamaged skin through various pathways, depending on the skin cell type.

In an additional effort to study the mechanism of improvement of photoaged skin by HA, Bernstein et al investigated the effects of glycolic acid on epidermal and dermal hyaluronic acid production and on the induction of collagen gene expression. ${ }^{23}$ They compared collagen gene expression from skin biopsy specimens and hyaluronic acid immunohistochemical staining from glycolic acid- and vehicle-treated skin. Sun-damaged forearm skin was treated with $20 \%$ glycolic acid lotion or a lotion vehicle control (oil in water; $\mathrm{pH}$ 3.9) twice a day for 3 months. The authors found that this protocol increased epidermal thickness, epidermal and dermal levels of hyaluronic acid, and collagen gene expression. Even small increases in the content of cutaneous hyaluronic acid may result in large changes in epidermal and dermal hydration, affecting skin appearance, texture, and function. With the growing concern regarding the photosensitivity aspects of HAs, this study documents some of the beneficial effects of glycolic acid.

Another proposed mechanism for the biological actions of HA was described by Rendl et al. ${ }^{6}$ They investigated the effects of creams containing lactic acid on the secretion of cytokines by keratinocytes in human reconstructed epidermis. ${ }^{6}$ They found that topically applied creams containing lactic acid $(1.5 \%, 3 \%$, or $5 \%)$ led to a concentration-dependent increase in apoptotic cells compared to the vehicle control. In addition, they found an increase in the secretion of vascular endothelial growth factor (VEGF) over the vehicle control after treatment with $1.5 \%$ or $3 \%$ lactic acid. No significant increase in VEGF secretion was detected following treatment with 5\% lactic acid. In contrast to this finding, the secretion of angiogenin was decreased by lactic acid in a concentrationdependent manner. No significant changes in IL-8 secretion were elicited by lactic acid at any of the concentrations tested. The authors concluded that topical application of lactic acid modulates the secretion of cytokines by keratinocytes and that this regulation might represent a mechanism contributing to their therapeutic effects such as photoaging. ${ }^{6}$

\section{SA and derivatives}

SA is widely used in cosmetic formulations (concentration $2 \%-4 \%$ ) and also therapeutically as a keratolytic (peeling) agent to treat skin conditions, such as calluses, keratoses, acne, and photoaging. It is applied in various concentrations, vehicles, and $\mathrm{pHs}$, depending on the numbers of lesions. Several experimental and clinical studies have found that topically applied SA is photoprotective. It has been reported that repeated topical application of SA or sodium salicylate protects SKH-1 mice from skin carcinogenesis induced by UV radiation. ${ }^{24} \mathrm{SA}$, applied to human skin shortly before exposure to UV radiation, was reported to decrease the erythemal response. ${ }^{25}$ One clinical investigation reported that SA (in a non-UV absorbing vehicle) had a pronounced filter effect when applied prior to UVB exposure. ${ }^{26} \mathrm{SA}$ added after UVB exposure had no measurable effect. The authors, therefore, discounted the contribution of the anti-inflammatory property of SA under the conditions of their experiment. In addition, Kristensen and Kristensen also performed a doubleblind clinical trial on patients with plaque psoriasis, ${ }^{26}$ and most subjects (73\%) cleared less on the side pretreated with $\mathrm{SA}$. The authors concluded that SA provides photoprotection of sufficient degree to warrant advising patients not to use SA 
concomitantly with UVB therapy. More recently, Birgin et al reported their results on the effect of topical petrolatum and SA $(20 \%)$ upon skin photoreaction to UVA. ${ }^{27}$ They concluded that the application of SA in petrolatum before PUVA therapy is not recommended because of its UVA-blocking effect.

The antibacterial action of SA has been known for many decades. One recent study indicates that SA acts at the level of transcription to downregulate the production of fibrinogen, fibronectin, and $\alpha$-hemolysin virulence factors necessary for bacterial replication in host tissues. Whether SA affects bacterial growth in acne was not reported in this study. ${ }^{28} \mathrm{~A} \mathrm{C}-8$ derivative of SA known as $\beta$-lipohydroxy acid ( $\beta$ LHA), developed in the late 1980s, has been proposed as an exfoliant and as a treatment of photoaged skin and acne. ${ }^{29} \beta$ LHA has an eight-carbon fatty chain linked to the benzene ring making it more lipophilic than SA. This may account for its slower skin penetration. $\beta$ LHA was shown to have a good safety profile with lower irritation when compared to glycolic acid. ${ }^{30} \beta \mathrm{LHA}$ also showed activity in reducing melanosome clustering and epidermal pigmentation. Contrary to $\alpha \mathrm{HAs}, \beta$ LHA increased the skin's resistance to UV-induced damage. ${ }^{30,31} \beta \mathrm{LHA}$ has antibacterial effects, which are ideal for the treatment of acne. ${ }^{30}$ Recently, Saint-Leger et al described in detail the properties of $\beta$ LHA, its cosmetic and clinical applications, and its biologic mechanism of action. ${ }^{32}$ The authors specifically discussed $\beta$ LHA's antimicrobial, anti-inflammatory, antifungal, and anticomedogenic properties. In summary, SA and its derivatives represent a rare case of a drug being used for over a century with no tendency to be replaced in the near future.

\section{PHAs}

As mentioned in the Introduction, PHAs and PHBAs represent the next generation of $\alpha H A$ s for use in cosmetic and dermatologic skin care. ${ }^{5}$ PHAs are $\alpha$ HAs containing multiple hydroxyl groups, while PHBAs are PHAs with an additional sugar molecule attached to the PHA structure. PHAs and PHBAs provide clinically proven antiaging and skin-smoothing effects that are comparable to $\alpha \mathrm{HAs}$, while offering several therapeutic advantages. In addition, these molecules function as humectants and moisturizers, as well as providing antioxidant chelation effects. ${ }^{5}$ The most popular PHA marketed today is gluconolactone (Figure 1). Bernstein et al conducted a study both in vitro and in a clinical setting, which demonstrated that gluconolactone can protect against the detrimental effects of UV radiation. ${ }^{33}$ They exposed fibroblast cultures obtained from the skin of transgenic mice to UVB, in the presence or absence of gluconolactone solutions. They demonstrated a dose-dependent protection of gluconolactone compared to the controls. As the UV absorption of gluconolactone is low, they concluded that its protective effect must be due to other mechanisms, such as its ability to function as a chelating agent and potency in scavenging free radicals. In the clinical part of that study, the authors treated 11 volunteers, with skin types I-III, with $8 \%$ glycolic acid lotion, with $8 \%$ gluconolactone lotion (lotion not specified), or with no treatment for 1 month (the effect of the lotion itself was not reported). After the last topical application of lotions, each site was irradiated with a dose of 1.5 MEDs from a SSR source. The glycolic acidtreated skin demonstrated a significant increase in sunburn cells, whereas the gluconolactone-treated site did not. ${ }^{33}$

\section{Effects of HAs on pigmentation}

An additional, much less explored property of HAs is their effect on pigmentation. $\alpha \mathrm{HAs}$, such as glycolic acid and lactic acid, have been reported to be effective in treating pigmentary lesions including melasma, solar lentigines, and postinflammatory hyperpigmentation. The proposed mechanism of this effect was believed to be due to epidermal remodeling and accelerated desquamation, which should result in quicker pigment dispersion. In 2003, Usuki et al published an in vitro study which showed that glycolic acid and lactic acid (300-500 $\mu \mathrm{g} / \mathrm{mL}$ ) suppressed melanin formation by directly inhibiting tyrosinase activity in human and mouse melanoma cells. ${ }^{34}$ They also reported that this effect was independent of the acidic nature of the HAs involved. Using their in vitro system, the authors conclude that glycolic acid and lactic acid might work not only by accelerating the epidermal turnover, but also by directly inhibiting melanin formation in melanocytes. If confirmed, their findings should have important clinical significance. The effect of topically applied HAs on tanning has been less examined. Tsai et al investigated the effects of glycolic acid on light-induced skin pigmentation in 6 Asian and in 6 Caucasian subjects. ${ }^{35}$ Subjects received UVA and UVB irradiations to both sides of the lower back and on the contralateral extensor forearms once daily for 7 days and then twice daily for 7 weeks. A placebo gel was applied to the opposite sides. They found increased UVB-induced tanning on the forearm and the lower back in both races in areas pretreated with glycolic acid. UVA caused increased tanning only on the exterior forearms in Asian subjects. The authors proposed that this small panel of subjects should be enlarged in future studies. To our knowledge, all these clinical studies were performed in subjects having skin types I-III. In order to address safety issues of populations with darker skin types, corresponding investigations with cohorts having skin types IV-VI are needed. 
Table I Applications of hydroxy acids

\begin{tabular}{|c|c|c|c|}
\hline Types/classes of HAs & Safety evaluation & Photocarcinogenesis & Mechanism of biological action \\
\hline$\alpha \mathrm{HAs}$ & $\begin{array}{l}\text { Not mutagenic or carcinogenic, } \\
\text { not reproductive or } \\
\text { developmental toxins, } \\
\text { not skin sensitizers }{ }^{\prime \prime}\end{array}$ & $\begin{array}{l}\text { No alteration of } \\
\text { photocarcinogenesis } \\
\text { induced by SSR }{ }^{13}\end{array}$ & $\begin{array}{l}\text { Reduced } \mathrm{Ca} \text { ion concentration in the epidermis } \\
\text { disrupts cellular adhesions by removing } \mathrm{Ca} \text { ions } \\
\text { from the cell adhesions by chelation allowing for } \\
\text { exfoliation; promoting cell growth and retarding } \\
\text { cell differentiation } 19\end{array}$ \\
\hline Glycolic acid & $\begin{array}{l}\text { Increased SSR sensitivity in } \\
\text { human skin }{ }^{9,10} \\
\text { Increased epidermal } \\
\text { thickness, epidermal and dermal } \\
\text { levels of hyaluronic acid, and } \\
\text { collagen gene expression }{ }^{23}\end{array}$ & $\begin{array}{l}\text { Increased epidermal } \\
\text { proliferation in mice }^{14}\end{array}$ & $\begin{array}{l}\text { Acceleration of collagen synthesis by fibroblasts } \\
\text { and also modulation of matrix degradation and } \\
\text { collagen synthesis through keratinocyte-released } \\
\text { cytokines }^{22} \\
\text { Accelerated epidermal turnover and inhibition } \\
\text { of melanin formation in melanocytes by directly } \\
\text { inhibiting tyrosinase activity }{ }^{34} \\
\text { Pretreatment increased UV-induced } \\
\text { pigmentation }{ }^{35}\end{array}$ \\
\hline $\mathrm{PHA}$ & & $\begin{array}{l}\text { Photoprotective } \\
\text { (gluconolactone) }^{33}\end{array}$ & $\begin{array}{l}\text { Function as a chelating agent and exhibits } \\
\text { potency in scavenging free radicals }{ }^{33}\end{array}$ \\
\hline$\beta \mathrm{HA}$ & - & - & - \\
\hline SA & $\begin{array}{l}\text { Enhances percutaneous penetration, } \\
\text { not a photosensitizer, not } \\
\text { phototoxic, no change in SSR } \\
\text { sensitivity in human skin } \\
\text { UV effects on skin sensitivity } \\
\text { should be considered due to its } \\
\text { keratolytic action on human skin }{ }^{12}\end{array}$ & $\begin{array}{l}\text { Photoprotective, reducing the } \\
\text { carcinogenicity of SSR }{ }^{13,24} \\
\text { Increased epidermal } \\
\text { proliferation and thickness } \\
\text { in mice }^{14}\end{array}$ & $\begin{array}{l}\text { Acts at the level of transcription to } \\
\text { downregulate the production of fibrinogen, } \\
\text { fibronectin, and } \alpha \text {-hemolysin virulence factors } \\
\text { necessary for bacterial replication } \\
\text { in host tissues }{ }^{28}\end{array}$ \\
\hline
\end{tabular}

\section{Cosmeceuticals}

Cosmeceuticals are cosmetic products that contain both cosmetic and biologically active ingredients purporting to have medical and/or drug-like benefits. The term is widely used not only in cosmetic advertising, but also in periodicals, monographs, and promotional materials. However, the Federal Food, Drug and Cosmetic Act does not recognize any such category as 'cosmeceuticals'. A product can be a cosmetic, a drug, or a combination of both, but the term 'cosmeceuticals' has no meaning under the law (see http:// www.fda.gov/Cosmetics/ProductandIngredientSafety/ PrductInformation/ucm127064.htm).

\section{Conclusion}

HAs have transformed approaches to skin care and have enjoyed huge success since their introduction to cosmetic and therapeutic dermatology about four decades ago. ${ }^{4}$ In spite of a large number of studies on HAs that have accumulated over this period, there is still an inadequate amount of information to assess the safety of topical application of these products. In many cases, it is difficult to compare the results of individual studies as they are performed under various conditions. The concentrations and $\mathrm{pHs}$ of the active ingredients, the timing of the application of the products, and most importantly, the composition of the control vehicle show significant variations. In studies involving UV-exposures, the light sources also differed significantly and were often not adequately defined. On the other hand, from all those studies, we have learned that topically applied HAs are capable of interacting with many basic biological processes that occur in mammalian skin (summarized in Table 1). They can affect and modify the processes of cell proliferation, cytokine excretion, and induction of apoptosis and can act as antioxidant/chelators, influence the skin barrier function, and act as moisturizers. Some investigators, therefore, still recommend that caution should be taken when using these products, in particular with their chronic use.

For further reading, the authors highly recommend the important contributions by van Scott et a ${ }^{36}$ and Green et al ${ }^{37}$ as well as a recently published monograph edited by Zoe Draelos. ${ }^{38}$ They contain a treasure of information on the subject as well as provide ideas for planning future studies on the safety of HAs.

\section{Acknowledgment}

This research was supported in part by the Intramural Research Program of the National Cancer Institute at NIH.

\section{Disclosure}

The authors report no conflicts of interest in this work. 


\section{References}

1. van Scott EJ, Yu RJ. Control of keratinization with alpha-hydroxy acids Arch Dermatol. 1974;110(4):586-590.

2. Jackson EM. AHA-type products proliferate in 1993. Cosmet Dermatol. $1993 ; 6: 22-26$.

3. Yu RJ, van Scott EJ. Alpha-hydroxyacids and carboxylic acids. J Cosmet Dermatol. 2004;3(2):76-87.

4. Green B. After 30 years ... the future of hydroxyacids. J Cosmet Dermatol. 2005;4(1):44-45.

5. Green BA, Briden E. PHAs and bionic acids: next generation of hydroxy acids. In: Zoe Diana Draelos, editor. Cosmeceuticals. 2nd ed. Amsterdam, The Netherlands: Elsevier Inc.; 2009:209-215.

6. Rendl M, Mayer C, Weninger W, Tschachler E. Topically applied lactic acid increases spontaneous secretion of vascular endothelial growth factor

7. Bernstein EF, Underhill CB, Lakkakorpi J, et al. Citric acid increases damaged skin. Dermatol Surg. 1997;23(8):689-694.

8. Yu RJ, van Scott EJ. Salicylic acid: not a b-hydroxy acid. Cosmetic Dermatol. 1997;10:27.

9. Kornhauser A, Wei RR, Yamaguchi Y, et al. The effects of topically applied glycolic acid and salicylic acid on ultraviolet radiation-induced erythema, DNA damage and sunburn cell formation in human skin. $J$ Dermatol Sci. 2009;55(1):10-17.

10. Kaidbey KH, Sutherland BM, Bennett P, et al. Topical glycolic acid enhances photodamage by ultraviolet light. Photodermatol Photoimmunol Photomed. 2003;19:21-27.

11. Andersen FA. Final report on the safety assessment of glycolic acid, ammonium, calcium, potassium, and sodium glycolates, methyl, ethyl, propyl, and butyl glycolates, and lactic acid, ammonium, calcium, potassium, sodium, and TEA-lactates, methyl, ethyl, isopropyl, and butyl lactates, and lauryl, myristyl, and cetyl lactates. Int J Toxicol. 1998;17 Suppl 1:1-241.

12. Andersen FA. Safety assessment of salicylic acid, butyloctyl salicylate, calcium salicylate, C12-15 alkyl salicylate, capryoyl salicylic acid, hexyldodecyl salicylate, isocetyl salicylate, isodecyl salicylate, magnesium salicylate, MEA-salicylate, ethylhexyl salicylate, potassium salicylate, methyl salicylate, myristyl salicylate, sodium salicylate, TEA-salicylate, and tridecyl salicylate. Int J Toxicol. 2003;22 Suppl 3:1-108.

13. National Toxicology Program Technical Report. Photocarcinogenic Study of Glycolic Acid and Salicylic Acid in SKH-1 Mice. Research Triangle Park, NC: National Toxicology Program; 2007:TR 524.

14. Sams RL, Couch LH, Miller BJ, et al. Basal cell proliferation in female SKH-1 mice treated with alpha- and beta-hydroxy acids. Toxicol Appl Pharmacol. 2001;175(1):76-82.

15. Sams RL, Couch LH, Miller BJ, et al. Effects of alpha- and beta-hydroxy acids on the edemal response induced in female SKH-1 mice by simulated solar light. Toxicol Appl Pharmacol. 2002;184:136-143.

16. Hong JT, Kim EJ, Ahn KS, et al. Inhibitory effect of glycolic acid on ultraviolet-induced skin tumorigenesis in SKH-1 hairless mice and its mechanism of action. Mol Carcinog. 2001;31(3):152-160.

17. Lu YP, Lou YR, Xie JG, et al. Tumorigenic effect of some commonly used moisturizing creams when applied topically to UVB-pretreated high-risk mice. J Invest Dermatol. 2009;129(2):468-475.

18. Forbes PD. Moisturizers, vehicle effects, and photocarcinogenesis. J Invest Dermatol. 2009;129(2):261-262. and related compounds. I. Topical treatment of ichthyotic disorders. by human reconstructed epidermis. Br J Dermatol. 2001;145(1):3-9. viable epidermal thickness and glycosaminoglycan content of sun-

19. Wang X. A theory for the mechanism of action of the alpha-hydroxy acids applied to the skin. Med Hypotheses. 1999;53(5):380-382.

20. Harris C, Livingstone S. Bidentate chelates. In: Dwyer F, Mellor D, editors. Chelating Agents and Metal Chelates. New York, NY: Academic Press; 1964:95-141.

21. Burge S. Cohesion in the epidermis. Br J Dermatol. 1994;131(2): 153-159.

22. Okano Y, Abe Y, Masaki H, Santhanam U, Ichihashi M, Funasaka Y. Biological effects of glycolic acid on dermal matrix metabolism mediated by dermal fibroblasts and epidermal keratinocytes. Exp Dermatol. 2003;12 Suppl 2:57-63.

23. Bernstein EF, Lee J, Brown DB, Yu R, van SE. Glycolic acid treatment increases type I collagen mRNA and hyaluronic acid content of human skin. Dermatol Surg. 2001;27(5):429-433.

24. Bair WB 3rd, Hart N, Einspahr J, et al. Inhibitory effects of sodium salicylate and acetylsalicylic acid on UVB-induced mouse skin carcinogenesis. Cancer Epidemiol Biomarkers Prev. 2002;11(12):1645-1652.

25. Weirich EG. Dermatopharmacology of salicylic acid. I. Range of dermatotherapeutic effects of salicylic acid. Dermatologica. 1975;151(5): $268-273$.

26. Kristensen B, Kristensen O. Topical salicylic acid interferes with UVB therapy for psoriasis. Acta Derm Venereol. 1991;71(1):37-40.

27. Birgin B, Fetil E, Ilknur T, Tahsin GA, Ozkan S. Effects of topical petrolatum and salicylic acid upon skin photoreaction to UVA. Eur J Dermatol. 2005;15(3):156-158.

28. Herrmann M. Salicylic acid: an old dog, new tricks, and staphylococcal disease. J Clin Invest. 2003;112(2):149-151.

29. Ditre M. Exfoliants, moisturizers and more: AHA's, BHA's and PHA's. In: Zoe Diana Draelos, editor. Cosmeceuticals. 2nd ed. Amsterdam, The Netherlands: Elsevier Inc.; 2009:111-119.

30. Pierard GE, Rougier A. Nudging acne by topical beta-lipohydroxy acid (LHA), a new comedolytic agent. Eur J Dermatol. 2002;12(4): XLVII-XLVIII.

31. Pierard GE, Nikkels-Tassoudji N, Arrese JE, Pierard-Franchimont C, Leveque JL. Dermo-epidermal stimulation elicited by a betalipohydroxyacid: a comparison with salicylic acid and all-trans-retinoic acid. Dermatology. 1997;194(4):398-401.

32. Saint-Leger D, Leveque JL, Verschoore M. The use of hydroxy acids on the skin: characteristics of C8-lipohydroxy acid. J Cosmet Dermatol. 2007;6(1):59-65.

33. Bernstein EF, Brown DB, Schwartz MD, Kaidbey K, Ksenzenko SM. The polyhydroxy acid gluconolactone protects against ultraviolet radiation in an in vitro model of cutaneous photoaging. Dermatol Surg. 2004; 30(2 Pt 1):189-195.

34. Usuki A, Ohashi A, Sato H, Ochiai Y, Ichihashi M, Funasaka Y. The inhibitory effect of glycolic acid and lactic acid on melanin synthesis in melanoma cells. Exp Dermatol. 2003;12 Suppl 2:43-50.

35. Tsai TF, Bowman PH, Jee SH, Maibach HI. Effects of glycolic acid on light-induced skin pigmentation in Asian and caucasian subjects. $J \mathrm{Am}$ Acad Dermatol. 2000;43(2 Pt 1):238-243.

36. van Scott EJ, Ditre CM, Yu RJ. Alpha-hydroxyacids in the treatment of signs of photoaging. Clin Dermatol. 1996;14(2):217-226.

37. Green BA, Yu RJ, van Scott EJ. Clinical and cosmeceutical uses of hydroxyacids. Clin Dermatol. 2009;27(5):495-501.

38. Zoe Diana Draelos, editor. Cosmeceuticals. 2nd ed. Amsterdam, The Netherlands: Elsevier Inc.; 2009

Clinical, Cosmetic and Investigational Dermatology

\section{Publish your work in this journal}

Clinical, Cosmetic and Investigational Dermatology is an international, peer-reviewed, open access, online journal that focuses on the latest clinical and experimental research in all aspects of skin disease and cosmetic interventions. All areas of dermatology will be covered; contributions will be welcomed from all clinicians and Submit your manuscript here: http://www.dovepress.com/clinical-cosmetic-and-investigational-dermatology-journal

\section{Dovepress}

basic science researchers globally. This journal is indexed on CAS The manuscript management system is completely online and includes a very quick and fair peer-review system, which is all easy to use. Visit http://www.dovepress.com/testimonials.php to read real quotes from published authors. 